\title{
Salvage Reconstructive Surgery in an Adult Patient with Failed Previous Repair of an Extrophy-Epispadias Complex. An Operation with a Functional and Aesthetic Purpose
}

\author{
Steven Joniau, Anne-Marie Stoel, Hein Van-Poppel, Robert Hierner
}

Department of Urology and Department of Plastic, Reconstructive and Aesthetic Surgery, Center for Interdisciplinary Reconstructive Surgery, Microsurgery, Hand Surgery, Burns, University Hospital Leuven, Leuven, Belgium

\begin{abstract}
Salvage surgical procedures after failed reconstruction for an extrophy-epispadias complex are extremely challenging. The goals are to restore continence and improve aesthetic appearance in order to provide quality of life and an improved body image to the patient.

We describe the surgical steps in an adult patient who presented anal urinary incontinence and a poor body image due to the absence of an umbilicus and the presence of hypertrophic scars. He underwent a modified Mainz II reconstruction of the lower urinary tract at childhood for an extrophy-epispadias complex. Restoration of continence was achieved by the construction of a modified Mainz I pouch with a continent stoma in a neo-umbilicus. Body image improved dramatically by the construction of a neo-umbilicus, a surgical revision of the hypertrophic abdominal scars and an abdominoplasty. It is mandatory that such demanding surgery should only be attempted as a combined multidisciplinary effort with urologists and plastic/reconstructive surgeons.
\end{abstract}

Key words: bladder exstrophy; epispadias; reconstructive surgical procedures

Int Braz. J Urol. 2007; 33: 810-4

\section{INTRODUCTION}

Children with the extrophy-epispadias complex pose a great challenge to the multidisciplinary teams caring for them. The main goals are to achieve urinary continence and fashioning the external genitalia in order to be capable of adequate sexual intercourse. At present, most teams will opt for a staged reconstruction with closure of the bladder plate, and a bilateral iliac osteotomy at a first stage. Later treatment is directed to render the patient continent, which often implies in the construction of urinary reservoirs and conduits for intermittent catheterization. An alternative is the construction of a rectal pouch (Mainz II procedure), with the anal sphincter providing continence. In addition, in this later stage, the external genitalia are reconstructed (1).

Although continence now can be achieved in up to $80 \%$ of children, only about $40 \%$ of adults are dry in the best hands. Apart from this, hypertrophic scars, the absence of an umbilicus and malformation of the external genitalia often compromise the aes- 
thetic aspects later in life. In these cases surgical procedures, which are complex and demanding, should be performed by multidisciplinary teams (1-5).

We describe the surgical steps undertaken in an adult patient who experienced secondary incontinence and a poor body image after failed previous surgery for an extrophy-epispadias complex. The main challenge was to restore continence and to create a neo-umbilicus, which served both a functional (access to the urinary reservoir) and aesthetic purpose.

\section{CASE REPORT}

A 35-year-old man presented at the urology outpatient clinic. As a neonate, he had been treated for extrophy-epispadias complex by the construction of a modified Mainz II pouch. The rectum served as a urinary reservoir, while the sigmoid colon was pulled down forming the posterior compartment. A corporoplasty, combined with a urethroplasty was performed at the same time. At the age of 14, a reimplantation of the left ureter was performed for an anastomotic stricture. A Nesbitt-plication and a scar revision were performed at the age of 29 . Furthermore, he underwent an inguinal hernia repair, an appendectomy.

Initially, continence was as expected in a rectal neo-bladder: normal during the day but at night there was some loss of urine. At the age of 32, the patient sustained a persistent transphincteric recto-perineal fistula. He was treated conservatively with a tressthread intending not to affect the function of the anal sphincter. In spite of good clinical practice, there was a partial loss of continence. A bulking agent was unsuccessfully implanted inter sphincter.

The main problems at presentation were a poor body image caused by the absence of the umbilicus and the broad, hypertrophic abdominal scars. The urinary incontinence was another main concern, as he had always been very active in different sports and had to stop these activities because of embarrassment with his situation.

At physical examination, wide and hypertrophic abdominal scars were evident, as was the absence of an umbilicus. The symphysis was widened because a pelvic osteotomy was not performed at childhood. The penis was short and broad.

Upper tract function was normal, with balanced split renal function on renal scintigraphy. There was no evidence of malignant malformation on rectoscopy. A retrograde urethrography showed a widened, saccular urethral remnant.

Considering the reconstructed urethra could not be catheterized and the fact that the umbilicus of the patient was missing as a result of the first operation, a neo-umbilicus had to be constructed to provide an aesthetic and functional access to the pouch. This was planned in two phases. First, an inverted T abdominoplasty was performed to remove old scars. (Figure-1). At the same time, an umbilicus was reconstructed by means of two lateral flaps. Woundhealing was compromised: there was an area of dehiscence of the vertical part of the scar. During the second stage, a redo umbilical reconstruction was performed simultaneously with the successful construction of continent iliocaecal neobladder (Mainz I-pouch) with a continent (Monti) stoma (post-appendectomy status), Figure-1. An additional liposuction was performed in order to improve the epigastric and lateral ("love handles") contour. After the second stage, wound healing was not compromised and the patient was taught to catheterize via the neo-umbilicus. At 12 months postoperatively, the patient was very pleased by both the functional and aesthetic results of the surgery. (Figure-1)

\section{COMMENTS}

The extrophy-epispadias complex is an uncommon malformation, characterized by disorders of the lower abdominal wall, pelvic floor, external genitalia and pelvis. Nowadays, staged reconstructions can achieve acceptable continence rates and aesthetic appearance. Nevertheless, a number of patients will fail, and will need additional surgery at a later stage. There are not many reports describing surgical steps to repair failed hypospadias surgery in adult patients $(1,2)$.

The presented case illustrates a number of considerations one has to make before embarking in such complex and challenging surgery. 

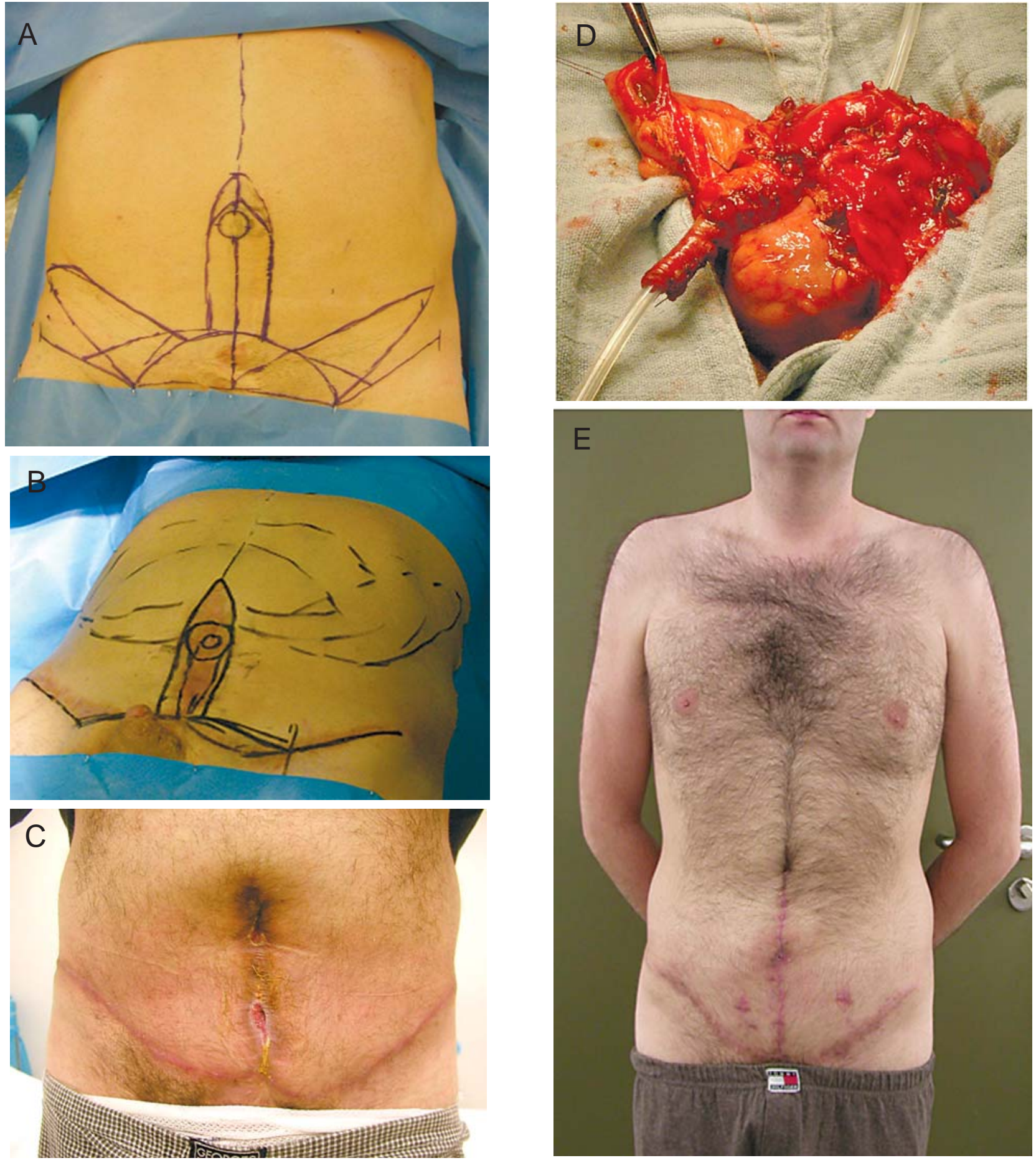

Figure 1-Construction of a functional neo-umbilicus in a 35-year-old man with previous bladder extrophy treatment. A) Clinical aspect: planning of the 1st stage operation (inverted T-abdominoplasty and neo-umbilicus construction). B) Clinical aspect 6 months after 1 st stage operation. C) Clinical aspect: planning of the 2 nd stage operation (liposuction and redo neo-umbilicus construction). D) Intraoperative aspect: modified Mainz I pouch with Monti type continent diversion. E) Clinical aspect 6 months after 2nd stage operation: aesthetic result. 

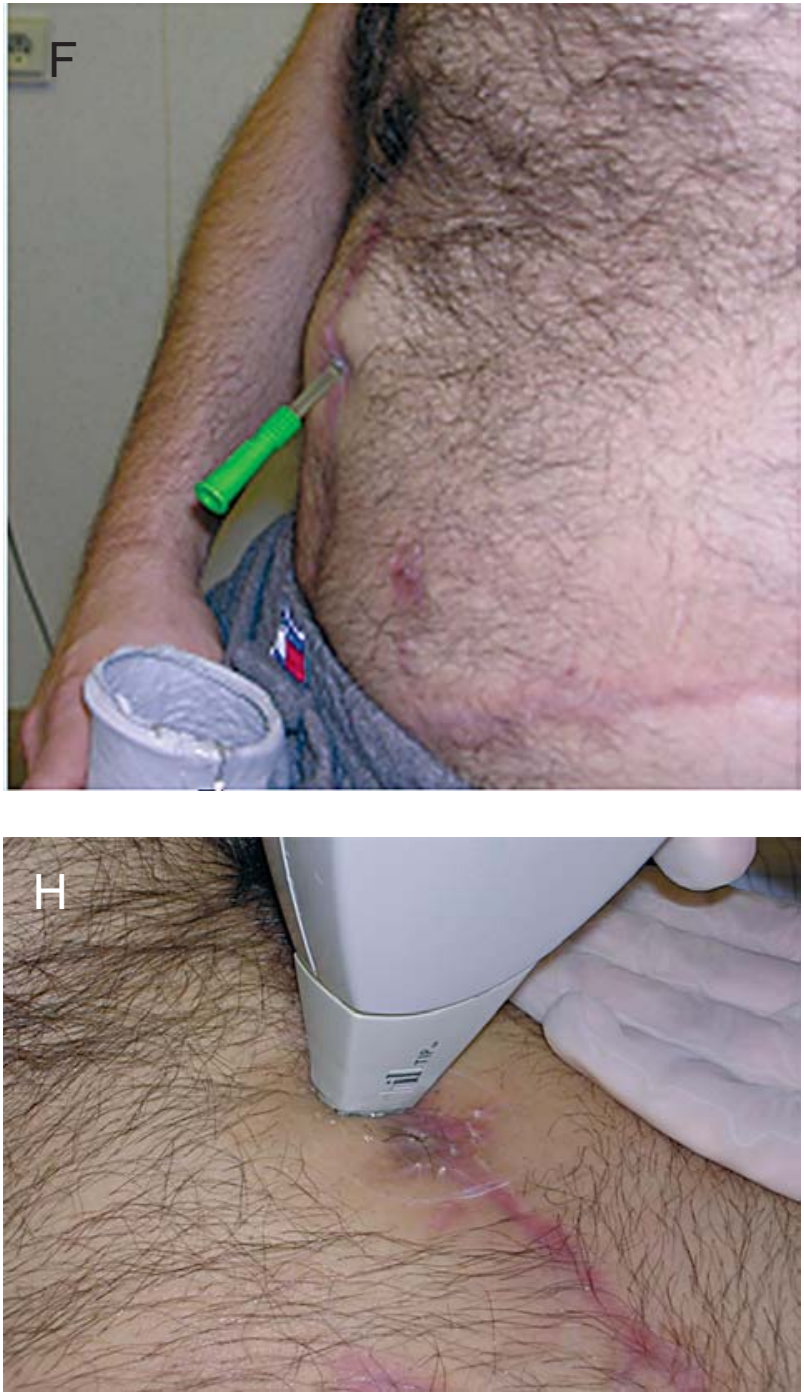

Secondary urinary incontinence after a previous rectal pouch reconstruction can only be treated by constructing a new urinary reservoir with a continent diversion from another bowel segment. In the presented case, a modified Mainz I pouch reconstruction was performed. A Monti type continent outlet was used, because the appendix had already been resected earlier in life. Reconstructive lower urinary tract surgery in secondary incontinent patients or patients remaining incontinent after prior surgery can achieve very high success rates, provided customized surgical procedures are chosen on a case-by-case basis (1).

The lack of the umbilicus was an important aesthetic burden for the patient. The absence of the

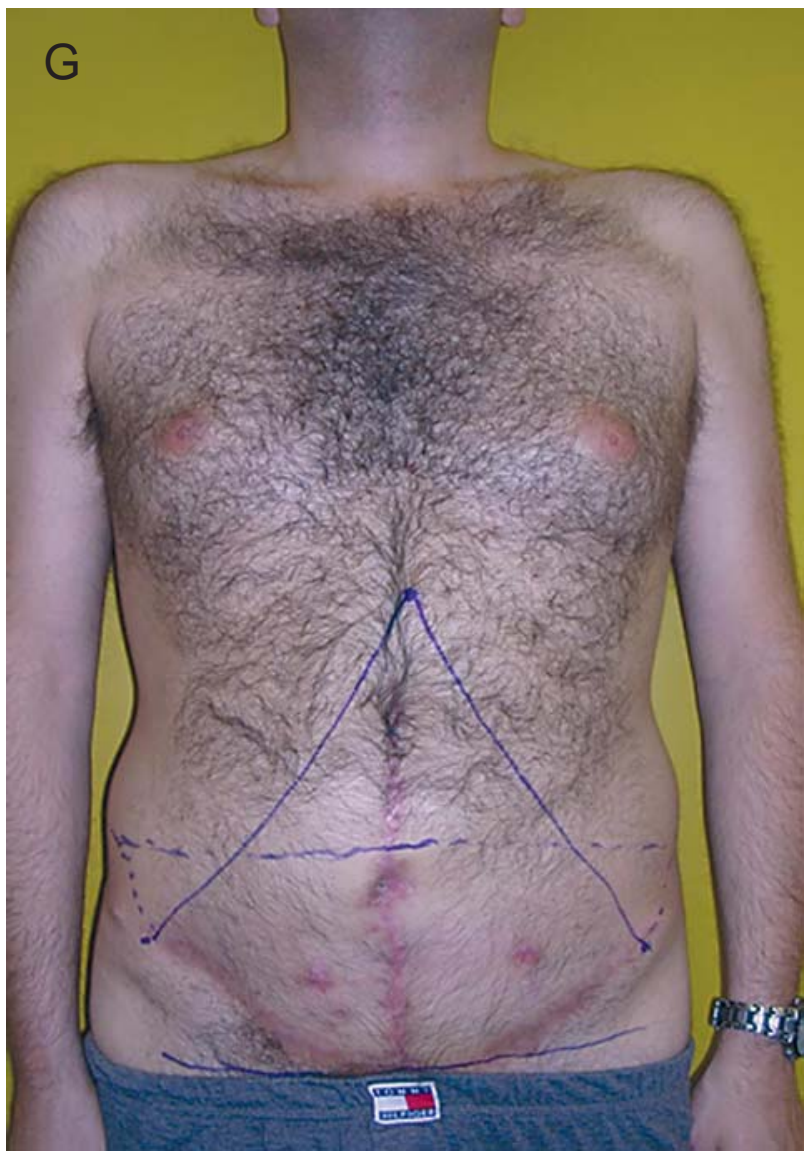

Figure 1 - continued - F) Clinical aspect 6 months after 2nd stage operation: functional result. G) Position of the neo-umbilicus. H) Laser epilation 6 months after 2nd stage operation: functional aspect.

only tolerated scar can be associated with poor body image and abnormal emotional behavior. Having not only an aesthetical function, the umbilicus can be used as a handy access for a continent diversion. Different techniques of umbilical reconstruction have been published, describing both the immediate reconstruction after primary closure of the bladder plate, as the delayed reconstruction at a later stage $(3,4)$.

In analogy with Choudhary \& Taams we suggest 4 details for functional and aesthetic reconstruction (5):

1) Preservation of the umbilicus: Contrary to old techniques, the umbilicus or even remnants should 
be preserved during primary treatment of bladder extrophy.

2) The position of the neo-umbilicus should be carefully planned: it should be placed on the joining lines of both iliac crests. The distance between the xiphisternum and the umbilicus - distance between the umbilicus and the pubic symphysis (1.6:1). - The distance between the umbilicus and the anterior superior iliac spine-distance between both the anterior superior iliac spines (0.6:1). The distance between the umbilicus and the anterior superior iliac spine-distance between the umbilicus and the pubic symphysis (0.9:1).

3) The appearance of the neo-umbilicus: The new umbilicus needs to have an adequate diameter (1.5 $-2 \mathrm{~cm}$ ). The navel is 'perfect' when he is T - or vertical shaped. The hooding should be different with the posture (superior in standing positioning). An 'innie' being better than an 'outie'. Better results are reached when the subcutaneous fat layer is more prominent. The natural cone depression must be pursued while a circumferentially scar should be avoided. Furthermore, it should have a normal slant superiorly and a normal shape.

4) The function of the neo-umbilicus: it should be continent and one should strive for minimal irritation at the transient zone between mucosa and normal skin. Adequate hygiene is of utmost importance in patients with hair growth. Hair follicles must be removed definitively by the exeresis or laser-treatment.

\section{CONCLUSION}

Body image, together with adequate urinary continence and sexual function are crucial in the surgical treatment of the extrophy-epispadias complex. When primary treatment fails at an adult age, surgery by an experienced multidisciplinary team can successfully improve patient's body image and restore continence.

\section{CONFLICT OF INTEREST}

None declared.

\section{REFERENCES}

1. Woodhouse CR, North AC, Gearhart JP: Standing the test of time: long-term outcome of reconstruction of the exstrophy bladder. World J Urol. 2006; 24: 244-9.

2. Meyer KF, Freitas Filho LG, Martins DM, Vaccari M, Carnevale J: The exstrophy-epispadias complex: is aesthetic appearance important? BJU Int. 2004; 93: 1062-8.

3. Barroso U Jr, Jednak R, Barthold JS, Gonzalez R: A technique for constructing an umbilicus and a concealed catheterizable stoma. BJU Int. 2001; 87: 117-20.

4. Hanna MK, Ansong K: Reconstruction of umbilicus in bladder exstrophy. Urology. 1984; 24: 324-6.

5. Choudhary S, Taams KO: Umbilicosculpture: a concept revisited. Br J Plast Surg. 1998; 51: 538-41. Erratum in: Br J Plast Surg 1999; 52: 78.

\author{
Correspondence address: \\ Dr. Steven Joniau \\ Dept. of Urology University Hospital Leuven \\ Herestraat 49 \\ 3000, Leuven, Belgium \\ E-mail: Steven.Joniau@uzleuven.be
}

